PROCEEDINGS OF THE

AMERICAN MATHEMATICAL SOCIETY

Volume 128, Number 1, Pages 189-194

S 0002-9939(99)04970-9

Article electronically published on June 21, 1999

\title{
ON THE MULTIPLICITIES OF THE ZEROS OF LAGUERRE-PÓLYA FUNCTIONS
}

\author{
JOE KAMIMOTO, HASEO KI, AND YOUNG-ONE KIM
}

(Communicated by Albert Baernstein II)

\begin{abstract}
We show that all the zeros of the Fourier transforms of the functions $\exp \left(-x^{2 m}\right), m=1,2, \ldots$, are real and simple. Then, using this result, we show that there are infinitely many polynomials $p\left(x_{1}, \ldots, x_{n}\right)$ such that for each $\left(m_{1}, \ldots, m_{n}\right) \in(\mathbb{N} \backslash\{0\})^{n}$ the translates of the function

$$
p\left(x_{1}, \ldots, x_{n}\right) \exp \left(-\sum_{j=1}^{n} x_{j}^{2 m_{j}}\right)
$$

generate $L^{1}\left(\mathbb{R}^{n}\right)$. Finally, we discuss the problem of finding the minimum number of monomials $p_{\alpha}\left(x_{1}, \ldots, x_{n}\right), \alpha \in A$, which have the property that the translates of the functions $p_{\alpha}\left(x_{1}, \ldots, x_{n}\right) \exp \left(-\sum_{j=1}^{n} x_{j}^{2 m_{j}}\right), \alpha \in A$, generate $L^{1}\left(\mathbb{R}^{n}\right)$, for a given $\left(m_{1}, \ldots, m_{n}\right) \in(\mathbb{N} \backslash\{0\})^{n}$.
\end{abstract}

\section{INTRODUCTION}

This paper is concerned with the zeros of real entire functions. Recall that a real entire function is an entire function which assumes only real values on the real axis. In [P2], G. Pólya proved that for each $m=1,2, \ldots$ the function $\psi_{m}(z)$ defined by

$$
\psi_{m}(z)=\int_{-\infty}^{\infty} \exp \left(-x^{2 m}\right) e^{i z x} d x \quad(z \in \mathbb{C})
$$

is a real entire function of order $\frac{2 m}{2 m-1}$ and has real zeros only;

$$
\psi_{1}(z)=\sqrt{\pi} \exp \left(-z^{2} / 4\right)
$$

has no zeros, and for $m \geq 2, \psi_{m}(z)$ has infinitely many zeros all of which are real. For generalizations of this result, see $[B]$. In $[K]$, the first author of this paper proved that all but a finite number of the zeros of $\psi_{m}(z)$ are simple, and conjectured that all the zeros of $\psi_{m}(z)$ are simple for $m=2,3, \ldots$.

In this paper, we will prove the following generalization of the conjecture.

Received by the editors February 2, 1998 and, in revised form, March 16, 1998.

1991 Mathematics Subject Classification. Primary 30D15, 30D35, 41A30, 43A20.

Key words and phrases. Fourier transform, Laguerre-Pólya function, Wiener's theorem.

The first author was partially supported by Grant-in-Aid for Scientific Research (No. 10740073), Ministry of Education, Science and Culture, Japan.

The second author was supported by Yonsei University Research Fund of 1998.

The third author was supported by the Korea Science and Engineering Foundation(KOSEF) through the Global Analysis Research Center(GARC) at Seoul National University. 
Theorem 1. For all $k=0,1,2, \ldots$ and $m=1,2, \ldots$ all the zeros of $\psi_{m}^{(k)}(z)$ are real and simple.

We will prove Theorem 1 in Section 2. In fact, we will prove a slightly more general one (Theorem 2). Our proof is based on special properties of the LaguerrePólya functions and the fact that each $\psi_{m}(z)$ satisfies a differential equation. Finally, in Section 3, we apply Theorem 1 to show that for each $n$-tuple $\left(m_{1}, \ldots, m_{n}\right)$ of positive integers and for each $n$-tuple of nonnegative integers $\left(k_{1}, \ldots, k_{n}\right)$ the translates of the function

$$
x_{1}^{k_{1}} \cdots x_{n}^{k_{n}}\left(1+x_{1}\right) \cdots\left(1+x_{n}\right) \exp \left(-\sum_{j=1}^{n} x_{j}^{2 m_{j}}\right)
$$

generate $L^{1}\left(\mathbb{R}^{n}\right)$, and conclude this paper with a discussion on the minimum number of monomials $p_{\alpha}\left(x_{1}, \ldots, x_{n}\right), \alpha \in A$, which have the property that the translates of the functions

$$
p_{\alpha}\left(x_{1}, \ldots, x_{n}\right) \exp \left(-\sum_{j=1}^{n} x_{j}^{2 m_{j}}\right) \quad(\alpha \in A)
$$

generate $L^{1}\left(\mathbb{R}^{n}\right)$, for a given $n$-tuple $\left(m_{1}, \ldots, m_{n}\right)$ of positive integers.

\section{The Laguerre-Pólya functions (Proof of Theorem 1)}

We start this section with a brief introduction to the Laguerre-Pólya functions. An entire function $\psi(z)$ is said to be a Laguerre-Pólya function if it can be expressed in the form

$$
\psi(z)=c z^{n} e^{-\alpha z^{2}+\beta z} \prod_{j}\left(1-\frac{z}{a_{j}}\right) e^{z / a_{j}},
$$

where $c, \beta, a_{j}$ are real, $\alpha \geq 0, n$ is a nonnegative integer and $\sum_{j}\left|a_{j}\right|^{-2}<\infty$. By a classical result of Laguerre [L] and Pólya [P1], an entire function $\psi(z)$ can be expressed in the form (2) if and only if there is a sequence of real polynomials with real zeros only which converges to $\psi(z)$ uniformly in compact sets in the complex plane. For a modern proof of this theorem, see Levin [Le, Chapter 8]. Therefore if $\psi(z)$ is a Laguerre-Pólya function, then all the derivatives of $\psi(z)$ are also LaguerrePólya functions. If $\psi(z)$ is given by (2), then the logarithmic derivative of $\psi(z)$ is given by

$$
\frac{\psi^{\prime}(z)}{\psi(z)}=\frac{n}{z}-2 \alpha z+\beta+\sum_{j}\left(\frac{1}{z-a_{j}}+\frac{1}{a_{j}}\right)
$$

and therefore

$$
\frac{d}{d z}\left(\frac{\psi^{\prime}}{\psi}\right)(z)<0 \quad(z \in \mathbb{R}, \psi(z) \neq 0),
$$

provided $\psi(z)$ is not of the form $\psi(z)=c e^{\beta z}$.

Let $\psi(z)$ be a transcendental Laguerre-Pólya function. If $a \in \mathbb{R}, \psi(a) \neq 0$ and $\psi^{\prime}(a)=0$, then $(3)$ implies that

$$
\psi(a) \psi^{\prime \prime}(a)<0
$$

in particular, $z=a$ is a simple zero of $\psi^{\prime}(z)$. Since all the derivatives of $\psi(z)$ are Laguerre-Pólya functions, we have the following. 
Proposition. Let $\psi(z)$ be a transcendental Laguerre-Pólya function. If $k$ is a positive integer and if $\psi^{(k)}(z)$ has a multiple zero at $z=a \in \mathbb{R}$, then

$$
\psi(a)=\psi^{\prime}(a)=\cdots=\psi^{(k)}(a)=0 .
$$

In particular, if $\psi(z)$ has simple zeros only, then all the derivatives of $\psi(z)$ have simple zeros only.

Remark 1. This proposition is closely related to the fact that a Laguerre-Pólya function has no Fourier critical points. For the definition of the Fourier critical points of real entire functions and related results, see [CCS], [KK], [Km2], [Km3], [P3]. It may also be remarked that if $\psi(z)$ is a transcendental Laguerre-Pólya function, then for each positive real number $B$ there is a positive integer $N$ such that $\psi^{(n)}(z)$ has only simple zeros in the interval $[-B \sqrt{n}, B \sqrt{n}]$ whenever $n \geq N$. For a proof of this fact, see $[\mathrm{Km} 1]$.

Now, consider the functions $\psi_{m}(z), m=1,2, \ldots$, defined by (1). It is clear that $\psi_{1}(z)=\sqrt{\pi} \exp \left(-z^{2} / 4\right)$ is a Laguerre-Pólya function. For $m \geq 2, \psi_{m}(z)$ is a real entire function of order less than 2 with real zeros only, and therefore Hadamard's theorem implies that $\psi_{m}(z)$ can be expressed in the form (2) with $\alpha=0$. Hence, to prove Theorem 1, it is enough to show that for each $m=1,2, \ldots, \psi_{m}(z)$ has simple zeros only, because of the proposition.

From an integration by parts, it can easily be shown that for each $m=1,2, \ldots$, $\psi_{m}(z)$ satisfies the differential equation

$$
\psi_{m}^{(2 m-1)}(z)-\frac{(-1)^{m}}{2 m} z \psi_{m}(z)=0 \quad(z \in \mathbb{C}) .
$$

Then Theorem 1 is a consequence of the following.

Theorem 2. Let $\psi(z)$ be a transcendental Laguerre-Pólya function, and assume that $\psi(z)$ satisfies the differential equation

$$
\psi^{(l)}(z)=A(z) \psi(z) \quad(z \in \mathbb{R})
$$

for some positive integer $l$ and some function $A(z)$ which is analytic in the whole real axis. Then all the (real) zeros of $\psi(z)$ are simple.

Proof. Assume, to get a contradiction, that $\psi(z)$ has a multiple zero, say at $z=$ $a \in \mathbb{R}$. Then $\psi(a)=\psi^{\prime}(a)=0$. From (5), we obtain $\psi^{(l)}(a)=0$. By differentiating both sides of $(5)$, we obtain

$$
\psi^{(l+1)}(z)=A^{\prime}(z) \psi(z)+A(z) \psi^{\prime}(z)
$$

so that $\psi^{(l+1)}(a)=0$. Then the proposition implies that

$$
\psi(a)=\psi^{\prime}(a)=\cdots=\psi^{(l)}(a)=\psi^{(l+1)}(a)=0 .
$$

By differentiating both sides of (5) $k(>0)$ times, we obtain

$$
\psi^{(l+k)}(z)=\sum_{\lambda=0}^{k}\left(\begin{array}{c}
k \\
\lambda
\end{array}\right) A^{(k-\lambda)}(z) \psi^{(\lambda)}(z) .
$$

Then (6), (7) and an inductive argument shows that

$$
\psi^{(k)}(a)=0
$$

for all $k=0,1,2, \ldots$, and this is the desired contradiction. 
Remark 2. Let $k$ and $l$ be integers with $0 \leq k<l$. If a transcendental LaguerrePólya function $\psi(z)$ satisfies the differential equation

$$
\psi^{(l)}(z)=A_{0}(z) \psi(z)+A_{1}(z) \psi^{\prime}(z)+\cdots+A_{k}(z) \psi^{(k)}(z) \quad(z \in \mathbb{R})
$$

for some functions $A_{0}(z), \ldots, A_{k}(z)$ which are analytic in the whole real axis, then a similar argument as in the above proof shows that every zero of $\psi(z)$ has multiplicity $\leq k+1$, or equivalently, all the zeros of $\psi^{(k)}(z)$ are simple.

\section{An Application to the haRmonic ANALYsis on $L^{1}\left(\mathbb{R}^{n}\right)$}

For a subset $\mathfrak{M}$ of the complex Banach algebra $L^{1}\left(\mathbb{R}^{n}\right)$ let $I(\mathfrak{M})$ denote the ideal generated by $\mathfrak{M}$, i.e., $I(\mathfrak{M})$ is the smallest closed linear subspace of $L^{1}\left(\mathbb{R}^{n}\right)$ which contains $\mathfrak{M}$, and has the property that

$$
f \in I(\mathfrak{M}) \text { and } g \in L^{1}\left(\mathbb{R}^{n}\right) \Rightarrow f * g \in I(\mathfrak{M}) .
$$

It is well known (see [R, Chapter 7]) that a closed linear subspace $V$ of $L^{1}\left(\mathbb{R}^{n}\right)$ is an ideal if and only if it is translation-invariant, i.e.,

$$
f \in V \Rightarrow f_{\mathbf{y}} \in V \quad\left(\mathbf{y} \in \mathbb{R}^{n}\right)
$$

where $f_{\mathbf{y}}$ is defined by

$$
f_{\mathbf{y}}(\mathbf{x})=f(\mathbf{x}+\mathbf{y}) \quad\left(\mathbf{x} \in \mathbb{R}^{n}\right)
$$

for $\mathbf{y} \in \mathbb{R}^{n}$. Therefore $I(\mathfrak{M})$ is the closed linear subspace of $L^{1}\left(\mathbb{R}^{n}\right)$ which is generated by the translates of the functions in the set $\mathfrak{M}$. The following theorem of $\mathrm{N}$. Wiener, whose proof can be found in [R, Chapter 7$]$ or in [W], gives a necessary and sufficient condition for a subset $\mathfrak{M}$ of $L^{1}\left(\mathbb{R}^{n}\right)$ to satisfy $I(\mathfrak{M})=L^{1}\left(\mathbb{R}^{n}\right)$ (or equivalently, the translates of all the functions in $\mathfrak{M}$ generate $L^{1}\left(\mathbb{R}^{n}\right)$ ).

Wiener's Theorem. Let $\mathfrak{M} \subset L^{1}\left(\mathbb{R}^{n}\right)$. Then $I(\mathfrak{M})=L^{1}\left(\mathbb{R}^{n}\right)$ if and only if there does not exist a point in $\mathbb{R}^{n}$ at which the Fourier transforms of all the functions in $\mathfrak{M}$ vanish simultaneously.

For $\mathbf{m}=\left(m_{1}, \ldots, m_{n}\right) \in(\mathbb{N} \backslash\{0\})^{n}$ let $\phi_{\mathbf{m}} \in L^{1}\left(\mathbb{R}^{n}\right)$ be defined by

$$
\phi_{\mathbf{m}}(\mathbf{x})=\exp \left(-\sum_{j=1}^{n} x_{j}^{2 m_{j}}\right) \quad\left(\mathbf{x}=\left(x_{1}, \ldots, x_{n}\right) \in \mathbb{R}^{n}\right) .
$$

Then Theorem 1 and Wiener's theorem imply that if $p(\mathbf{x})=x_{1}^{k_{1}} \cdots x_{n}^{k_{n}}\left(1+x_{1}\right) \cdots$ $\left(1+x_{n}\right)$, where $k_{1}, \ldots, k_{n}$ are nonnegative integers, then

$$
I\left(\left\{p(\mathbf{x}) \phi_{\mathbf{m}}(\mathbf{x})\right\}\right)=L^{1}\left(\mathbb{R}^{n}\right)
$$

for each $\mathbf{m} \in(\mathbb{N} \backslash\{0\})^{n}$.

Let $\mathbf{m}=\left(m_{1}, \ldots, m_{n}\right) \in(\mathbb{N} \backslash\{0\})^{n}$ be given. In the remainder of this paper, we will be interested in the minimum number $M(\mathbf{m})$ of monomials $p_{\alpha}(\mathbf{x}), \alpha \in A$, which satisfy

$$
I\left(\left\{p_{\alpha}(\mathbf{x}) \phi_{\mathbf{m}}(\mathbf{x}): \alpha \in A\right\}\right)=L^{1}\left(\mathbb{R}^{n}\right) .
$$

Assume, for a moment, that $m_{j} \geq 2$ for all $j=1, \ldots, n$. If $p(\mathbf{x})=x_{1}^{k_{1}} \cdots x_{n}^{k_{n}}$ is a monomial, then the Fourier transform of $p(\mathbf{x}) \phi_{\mathbf{m}}(\mathbf{x})$ is

$$
\prod_{j=1}^{n}(-i)^{k_{j}} \psi_{m_{j}}^{\left(k_{j}\right)}\left(z_{j}\right)
$$


Therefore if we are given a set $\left\{p_{\alpha}(\mathbf{x}): \alpha \in A\right\}$ of monomials, then the set of Fourier transforms of the functions $p_{\alpha}(\mathbf{x}) \phi_{\mathbf{m}}(\mathbf{x}), \alpha \in A$, can be written in the form

$$
\left\{f_{\alpha, 1}\left(z_{1}\right) f_{\alpha, 2}\left(z_{2}\right) \cdots f_{\alpha, n}\left(z_{n}\right): \alpha \in A\right\},
$$

where each $f_{\alpha, j}$ has infinitely many real zeros for $\alpha \in A$ and $j=1, \ldots, n$. If $\# A \leq n$, then it is clear that there is a point in $\mathbb{R}^{n}$ at which all the functions in the above set vanish simultaneously. Consequently, we have

$$
n+1 \leq M(\mathbf{m}) .
$$

For $\mathbf{k}=\left(k_{1}, \ldots, k_{n}\right) \in\{0,1\}^{n}$ let $p_{\mathbf{k}}(\mathbf{x})=x_{1}^{k_{1}} \cdots x_{n}^{k_{n}}$. Then it is easy to see that there does not exist a point in $\mathbb{R}^{n}$ at which the Fourier transforms of all the functions in the set

$$
\left\{p_{\mathbf{k}}(\mathbf{x}) \phi_{\mathbf{m}}(\mathbf{x}): \mathbf{k} \in\{0,1\}^{n}\right\}
$$

vanish simultaneously, so that

$$
M(\mathbf{m}) \leq 2^{n} .
$$

In the general case, we have

$$
r(\mathbf{m})+1 \leq M(\mathbf{m}) \leq 2^{r(\mathbf{m})} \quad\left(\mathbf{m}=\left(m_{1}, \ldots, m_{n}\right) \in(\mathbb{N} \backslash\{0\})^{n}\right),
$$

where $r(\mathbf{m})$ denotes the number of the indices $j$ for which $m_{j} \geq 2$.

Finally, we consider an improvement of the inequality (8). The inequality follows from the fact that for each $m=1,2, \ldots$ there are nonnegative integers $k_{1}$ and $k_{2}$, namely 0 and 1 , such that the zero sets of $\psi_{m}^{\left(k_{1}\right)}(z)$ and $\psi_{m}^{\left(k_{2}\right)}(z)$ are disjoint. In fact, for each $m=1,2, \ldots$ the zero sets of the functions $\psi_{m}(z), \psi_{m}^{\prime}(z)$ and $\psi_{m}^{(2 m)}(z)$ are mutually disjoint, because of the differential equation

$$
\psi_{m}^{(2 m)}(z)-\frac{(-1)^{m}}{2 m} z \psi_{m}^{\prime}(z)-\frac{(-1)^{m}}{2 m} \psi_{m}(z)=0 \quad(z \in \mathbb{C}),
$$

which is obtained by differentiating both sides of (4). Hence there does not exist a point in $\mathbb{R}^{n}$ at which the Fourier transforms of all the functions in the set

$$
\begin{aligned}
\left\{p\left(x_{1}, x_{2}\right) x_{3}^{k_{3}} \cdots x_{n}^{k_{n}} \phi_{\mathbf{m}}(\mathbf{x}): p\left(x_{1}, x_{2}\right)=1, x_{1} x_{2}, \text { or } x_{1}^{2 m_{1}} x_{2}^{2 m_{2}},\right. & \text { and } \left.k_{3}, \ldots, k_{n} \in\{0,1\}\right\}
\end{aligned}
$$

vanish simultaneously, so that $M(\mathbf{m}) \leq 3 \cdot 2^{n-2}$ for $n \geq 2$. Therefore we can replace the inequality $M(\mathbf{m}) \leq 2^{r(\mathbf{m})}$ in (9) by $M(\mathbf{m}) \leq 3 \cdot 2^{r(\mathbf{m})-2}$ whenever $r(\mathbf{m}) \geq 2$.

Let $l$ be an integer greater than 3 . If it were true that for each $m=1,2, \ldots$ there are nonnegative integers $k_{1}, \ldots, k_{l}$ such that the zero sets of $\psi_{m}^{\left(k_{1}\right)}(z), \ldots, \psi_{m}^{\left(k_{l}\right)}(z)$ are mutually disjoint, then a similar argument as above would imply that $M(\mathbf{m})=$ $r(\mathbf{m})+1$ if $r(\mathbf{m}) \leq l-1$, and $M(\mathbf{m}) \leq l \cdot 2^{r(\mathbf{m})-l+1}$ if $r(\mathbf{m})>l-1$. We do not know whether or not such nonnegative integers exist, so we conclude this paper with the following question.

Question. Let $l \geq 4$ and $m \geq 1$ be integers. Do there exist nonnegative integers $k_{1}, \ldots, k_{l}$ such that the zero sets of $\psi_{m}^{\left(k_{1}\right)}(z), \ldots, \psi_{m}^{\left(k_{l}\right)}(z)$ are mutually disjoint? 


\section{ACKNOWLEDGMENT}

The first author would like to thank Professor Katsunori Iwasaki for teaching him the relationship between the order of zeros of some Fourier integrals and the harmonic analysis on $L^{1}\left(\mathbb{R}^{n}\right)$. The authors thank Professor Hyeonbae Kang for informing the third author of the first author's conjecture. Finally, the authors thank the referee for his kind suggestions.

\section{REFERENCES}

[B] N. G. de Bruijn, The roots of trigonometric integrals, Duke Math. J. 17 (1950), 197-226. MR 12:250a

[CCS] T. Craven, G. Csordas and W. Smith, The zeros of derivatives of entire functions and the Pólya-Wiman conjecture, Ann. of Math. (2) 125 (1987), 405-431. MR 88a:30007

[K] J. Kamimoto, On an integral of Hardy and Littlewood, Kyushu J. of Math. 52 (1998), 249-263. CMP 98:09

[KK] H. Ki and Y. O. Kim, Proof of the Fourier-Pólya conjecture, preprint.

[Km1] Y. O. Kim, A proof of the Pólya-Wiman conjecture, Proc. Amer. Math. Soc. 109 (1990), 1045-1052. MR 90k:30049

[Km2] Critical points of real entire functions and a conjecture of Pólya, Proc. Amer. Math. Soc. 124 (1996), 819-830. MR 96f:30027

[Km3] Critical points of real entire functions whose zeros are distributed in an infinite strip, J. Math. Anal. Appl. 204 (1996), 472-481. MR 98e:30030

[L] E. Laguerre, Oeuvres I, Gauthier-Villars, Paris, 1898.

[Le] B. Ja. Levin, Distribution of Zeros of Entire Functions, Transl. Math. Mono., vol. 5, A.M.S., Providence, R.I., 1964. MR 81k:30011

[P1] G. Pólya, Über Annäherung durch Polynome mit lauter reellen Wurzeln, Rend. Circ. Mat. Palermo 36 (1913), 279-295.

[P2] _ On the zeros of an integral function represented by Fourier's integral, Messenger of Math. 52 (1923), 185-88.

[P3] - Some problems connected with Fourier's work on transcendental equations, Quart. J. Math. Oxford Ser. 1 (1930), 21-34.

[R] W. Rudin, Fourier Analysis on Groups, Interscience Publishers, 1962. MR 27:2808

[W] N. Wiener, Tauberian theorems, Ann. of Math. 33 (1932), 1-100.

Department of Mathematics, Kumamoto University, Kumamoto 860, Japan

E-mail address: joe@sci.kumamoto-u.ac.jp

Department of Mathematics, Yonsei University, Seoul 120-749, Korea

E-mail address: haseo@bubble.yonsei.ac.kr

Department of Mathematics, Sejong University, Seoul 143-747, Korea

E-mail address: kimyo@kunja.sejong.ac.kr 